\title{
Contribution à une approche renouvelée des statistiques sur l'emploi touristique
}

Contribution to a renewed approach to the tourist employment statistics

\section{Philippe Violier}

\section{(2) OpenEdition}

\section{Journals}

Édition électronique

URL : http://journals.openedition.org/tourisme/2322

DOI : 10.4000/tourisme.2322

ISSN : 2492-7503

\section{Éditeur}

Éditions touristiques européennes

\section{Référence électronique}

Philippe Violier, «Contribution à une approche renouvelée des statistiques sur l'emploi touristique », Mondes du Tourisme [En ligne], 16 | 2019, mis en ligne le 01 décembre 2019, consulté le 09 avril 2020. URL : http://journals.openedition.org/tourisme/2322 ; DOI : https://doi.org/10.4000/tourisme.2322

Ce document a été généré automatiquement le 9 avril 2020.

\section{c)}

Mondes du tourisme est mis à disposition selon les termes de la licence Creative Commons Attribution - Pas d'Utilisation Commerciale - Pas de Modification 4.0 International. 


\title{
Contribution à une approche renouvelée des statistiques sur l'emploi touristique
}

\author{
Contribution to a renewed approach to the tourist employment statistics
}

Philippe Violier

1 Cette analyse s'inscrit à la suite d'une réflexion sur le dénombrement des « voyageurs » internationaux selon l'Organisation mondiale du tourisme (OMT) et les institutions gouvernementales publiée récemment (Stock, Coeffé et Violier, 2017). Dans le même ordre d'idées, des données sont mobilisées au sujet des emplois touristiques. Ainsi, le global des emplois en France est estimée à +/-10\%. L'effectif salarié dans le secteur serait de l'ordre de $8 \%$ du total (1 038559 sur 12839 130) selon le Mémento du tourisme 2017 (données de 2016), publication annuelle de la direction générale des Entreprises du ministère de l'Économie et des Finances. Les recherches sur cette question dans la littérature scientifique s'appuient sur les données produites par les organismes institutionnels. Si une distance est parfois prise (Clergeau, 2013), nous relevons que cet angle de l'emploi est peu travaillé (Raboteur, 2000), quand le sujet n'est pas seulement effleuré (Sinclair et Stabler, 1997 ; Caccomo, 2007 ; Vellas, 2007 ; Botti et al., 2013). Nous nous proposons donc, dans cet article, de nous interroger tout d'abord de manière plus précise sur les conditions dans lesquelles ces données sont produites; nous montrerons ainsi les biais qui perturbent l'analyse. Puis, nous esquisserons une piste de recherche pour établir des données plus robustes.

\section{Une combinaison entre choix par défaut et raisonnement économique}

2 Les données sur l'emploi en France sont élaborées par deux organismes institutionnels. D'une part, l'Insee a publié en 2015 un numéro d'Insee Première consacré à l'emploi touristique local, soit les emplois « directement liés à la présence de touristes sur le lieu 
de leur séjour » (Durieux et al., 2015, p. 1). Ce choix met de côté, de fait, le secteur des transports ainsi que celui des agences de voyage, mais confond le tourisme et les voyages d'affaires. D'autre part, le Mémento du tourisme, publié par la direction générale des Entreprises aborde également la question des emplois, notamment dans sa dernière édition (2018, chiffres de 2017). L'analyse de ces deux publications permet d'informer plusieurs critiques.

Premièrement, la base de la définition d'un secteur du «tourisme " s'inscrit dans la comptabilité nationale, dont l'objet consiste à mesurer la valeur ajoutée produite par l'économie nationale. Elle vise de ce fait à éviter les doubles comptes provenant des chevauchements possibles entre différentes branches. Ainsi, les déplacements des "touristes» qui animent ce secteur économique à côté de ceux effectués par les habitants ne sont pas pris en compte, puisqu'ils sont déjà inclus dans la branche transport. La branche du "tourisme» est ainsi construite à partir des "activités caractéristiques ", appellation qui couvre un ensemble hétérogène dont l'unité ne tient qu'au fait qu'aucune autre branche de la comptabilité nationale ne les englobe. C'est donc un choix par défaut. Afin cependant de ne pas minimiser les effets économiques du «tourisme », l'OMT a recommandé l'usage du compte satellite du tourisme (CST) qui, à côté des activités caractéristiques, évalue la contribution du secteur aux autres branches et permet de mesurer la consommation touristique intérieure des résidents et des non-résidents, en France.

4 Or parmi les branches du "tourisme", trois d'entre elles rassemblent $89 \%$ des emplois : les « hébergements touristiques » pour $17 \%$, les « restaurants et cafés » pour $49 \%$ et les « services non urbains de transport » pour $23 \%$.

5 Si les entreprises de l'hébergement peuvent être, en grande partie, considérées comme relevant des mobilités, toutes ces dernières relèvent-elles du tourisme au sens strict? Nous touchons là à une question fondamentale qui partage les chercheurs: les uns, pour délimiter le tourisme, s'approprient la définition en usage dans les institutions et défendue par l'OMT ${ }^{1}$; les autres défendent une approche plus restrictive, soit un système qui a pour finalité la recréation des individus, ce qui exclut de fait les voyages d'affaires, les pèlerinages et autres voyages scolaires (Knafou et Stock, 2003). Ces auteurs insistent sur le fait que le tourisme est une mobilité du temps libre, qui place les individus au centre des choix essentiels (choix de la destination, des compagnes et compagnons de voyage, de l'usage du temps sur place...; alors que dans les voyages d'affaires, les décisions relèvent de la stratégie de l'entreprise, y compris pour les voyages dits de stimulation, de récompense ou autres) (Stock et al., 2018).

6 C'est également loin d'être le cas des restaurants et des cafés, qui sont également fréquentés par les habitants. Les proportions de non-résidents, dont les touristes, et d'habitants au sein de leur clientèle sont variables selon les lieux. La DGE le reconnaît d'ailleurs :

Les indicateurs d'activité, d'emplois et de démographie d'entreprises mentionnés portent sur l'ensemble des secteurs liés au tourisme, sachant qu'une partie seulement de ces secteurs, variable selon les cas, relève effectivement du tourisme. Ainsi, par exemple, le total des emplois ne concerne pas que l'emploi touristique, mais la totalité de l'emploi dans les secteurs caractéristiques du tourisme. (DGE, 2017, p. 23) 
Or cette précaution, prise dans l'ouvrage, est systématiquement oubliée dans les communications ultérieures, notamment lorsque sont proclamées les données sur l'emploi touristique.

Deuxièmement, ce choix est aussi inspiré par le modèle de la « chaîne de valeur » et par une définition économiciste du tourisme, qui réduit le tourisme à un échange marchand, alors que la part du non-marchand est importante. Selon les statistiques de la DGE elle-même, les personnes qui résident en France et qui passent une nuit en dehors de leur résidence principale, dans le cadre de voyages personnels, ont eu recours en 2017, pour 62,4 \% des nuitées, à des hébergements non marchands ${ }^{2}$. Là encore, la part du tourisme est difficile à apprécier au sein de cette catégorie des " voyages personnels » car elle englobe, outre le tourisme au sens strict, les visites aux parents et amis qui relèvent des relations sociales et revêtent donc un caractère obligatoire du point de vue des normes sociales. Mais le non-marchand génère des activités marchandes : bâtiment, construction, entretien paysagiste, alimentation... Ces dépenses sont prises en compte dans le CST au titre des dépenses des touristes.

Troisièmement, les personnels des organisations institutionnelles ne sont pas pris en compte dans ce décompte des emplois. Or, le réseau "Tourisme \& territoires », qui anime les agences départementales, revendique sur son site 2000 emplois $^{3}$. Il conviendrait également d'ajouter les emplois occupés au sein des structures locales et intercommunales, ainsi qu'au niveau régional.

10 Ensuite, dans la publication de la DGE les données incluent les services des agences de voyage et voyagistes, alors que l'Insee (Durieux et al., 20015) les exclue en se concentrant sur « l'emploi local ». Certes, l'activité des agences de voyage - appellation qui recouvre aussi bien les agences de voyage au sens strict, qui sont des points de vente, que les tour-opérateurs qui élaborent les prestations, circuits et séjours distribués par les premières - est en grande partie orientée vers l'outgoing. Mais une partie de l'activité de cet ensemble est au contraire orientée vers l'accueil in situ et relève donc bien de l'emploi local. Certaines entreprises notamment, les agences réceptives, sont totalement orientées vers cette fonction.

11 Enfin, outre le flou qui entoure la définition du périmètre du secteur et donc des emplois qui en relèvent, l'analyse longitudinale est également perturbée par des modifications fréquentes. En nous basant sur trois éditions du Mémento du tourisme, nous avons pu établir le tableau suivant, qui met en évidence les modifications apportées. La colonne de droite indique les observations que nous inspire cette approche longitudinale. Nous avons retenu trois années : 2009 comme année de base pour conduire les observations ; 2013 car il n'y a pas de changement entre 2009 et 2013 ; et enfin 2015. Des catégories sont apparues en 2013 et 2015. Certaines ont vu leur périmètre redéfini. Une autre a été mentionnée en marge du tableau en 2009 . Une dernière a disparu en 2013. Notons cependant qu'elles portent sur des effectifs réduits.

Tableau 1. Évolution des catégories statistiques prises en compte dans le Mémento du tourisme, publié annuellement par la DGE

\begin{tabular}{|l|l|l|l|}
\hline & 2009 & 2013 & 2015 \\
\hline
\end{tabular}




\begin{tabular}{|c|c|c|c|}
\hline $\begin{array}{l}\text { Apparition de } \\
\text { nouvelles catégories }\end{array}$ & & $\begin{array}{l}\text { Sites et monuments } \\
\text { historiques } \\
\text { (2 } 956 \text { emplois) }\end{array}$ & $\begin{array}{l}\text { Organisation de jeux de } \\
\text { hasard et d'argent } \\
\text { (17 } 528 \text { emplois) }\end{array}$ \\
\hline $\begin{array}{ll}\text { Évolution du } & \mathrm{du} \\
\text { périmètre } & \mathrm{de} \\
\text { certaines catégories }\end{array}$ & & $\begin{array}{l}\text { Activités récréatives } \\
\text { (7 } 834 \text { emplois) }\end{array}$ & $\begin{array}{l}\text { Parcs d'attraction et } \\
\text { autres services récréatifs } \\
\text { (31 } 849 \text { emplois) }\end{array}$ \\
\hline $\begin{array}{l}\text { Catégories } \\
\text { mentionnées en } \\
\text { marge }\end{array}$ & $\begin{array}{l}\text { Location de courte } \\
\text { durée de voitures... } \\
\text { (11 } 491 \text { emplois) }\end{array}$ & & \\
\hline $\begin{array}{l}\text { Disparition de } \\
\text { catégories }\end{array}$ & & $\begin{array}{l}\text { Entretien corporel } \\
\text { (9 } 071 \text { emplois en 2009) }\end{array}$ & \\
\hline
\end{tabular}

\section{Position de recherche : inverser le modèle}

Il est prématuré de proposer une méthode alternative. Nous pouvons seulement inviter à engager une réflexion qui, au lieu de définir a priori un ensemble de secteurs et en se fondant sur un concept robuste du tourisme, partirait des pratiques réelles des individus touristes qui génèrent des dépenses auprès d'entreprises et d'organisations qui pourraient dès lors être considérées comme relevant du tourisme, dans une proportion à définir. L'emploi en serait ensuite déduit. La démarche consiste à identifier, en premier lieu, les pratiques des touristes. Ensuite, nous mobilisons la typologie des lieux, selon le principe que, parmi les lieux touristiques, certains sont spécialisés et donc une grande partie de leurs emplois dépendent du tourisme, tandis que dans d'autres, polyfonctionnels, seule une partie, à déterminer, des emplois peut être qualifiée de touristique. Il serait enfin nécessaire d'introduire une dimension de saisonnalité.

\subsection{Les comportements des touristes}

13 La première étape consiste à identifier des contextes touristiques types à partir, d'une part, des modes d'accès aux lieux touristiques pratiqués par les individus. Nous proposons de mobiliser une modélisation (schéma 2) déjà publiée (Stock et al., 2018). 
Schéma 2. Position de recherche à partir d'un modèle économique/chaîne de valeur alternatif

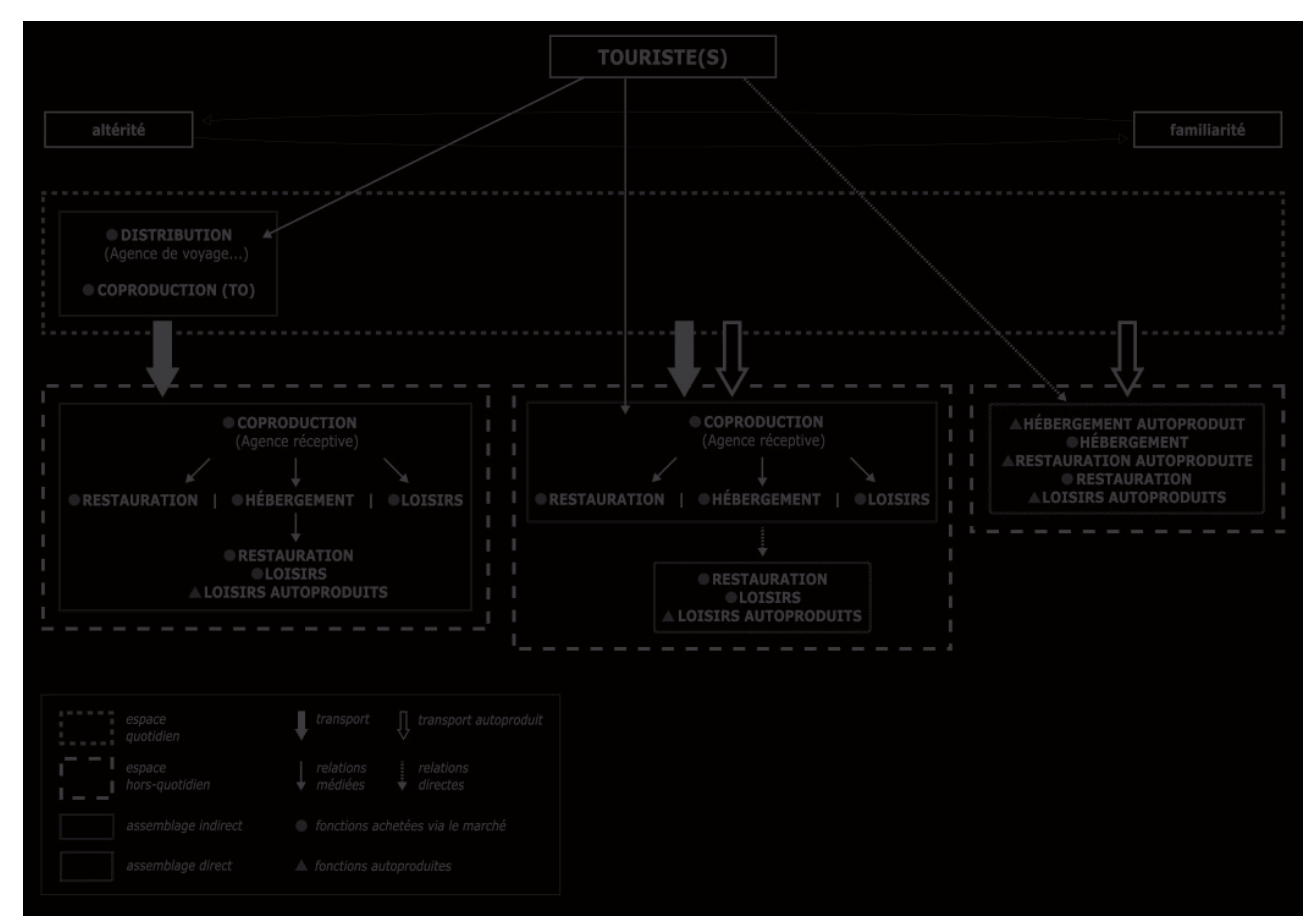

Source : élaboré par l'auteur, publié dans Stock et al., 2018

14 La modélisation que nous proposons repose sur une analyse des pratiques qui oppose deux situations extrêmes. D'une part, les individus sont confrontés, pour mettre en œuvre leur projet touristique, à une forte altérité objective, c'est-à-dire que des horizons d'altérité peuvent être définis (langue, culture, manières d'être, mal être corporel...) résultant de l'hétérogénéité culturelle ou biophysique du monde. Pour affronter cette altérité, les individus sollicitent des technologies spatiales (Stock, 2008), telles que le voyage organisé tout compris pour une pratique de découverte ou le séjour en hôtel-club dans le cas d'une recherche de repos ou de jeux. La chaîne de valeur (agent de voyage-tour opérateur-transporteur-hébergeur) fonctionne. Dans ces situations il est possible de considérer que la très grande partie des emplois est strictement cantonnée aux entreprises caractéristiques du tourisme.

Dans le détail, cependant, deux nuances peuvent être apportées. D’une part, la chaîne de valeur est concurrencée par des médiations opérées par des relations sociales (parents et amis) qui offrent aux individus des alternatives. D'autre part, sur leur lieu de destination, les individus trouvent des marges de manœuvre dans l'articulation entre les établissements contrôlés par les grands groupes capitalistes, inclus dans la chaîne de valeur, et les artisans locaux qui gravitent à proximité et qui n'y sont pas intégrés. Par exemple, à Maurice, l'expérience de nager avec les dauphins est essentiellement proposée dans la Baie de Tamarin par de petites entreprises locales, alors qu'une grande part de la fréquentation de l'île reste contrôlée par les grands groupes maîtrisant les hôtels-clubs.

16 À l'opposé, en situation de forte familiarité, les touristes entrent en relation directe, ou non intermédiée, avec des entreprises variées. C'est notamment le cas lorsque des individus qui résident en France métropolitaine y déploient également leurs pratiques touristiques. Or ce cas de figure est dominant: en 2017, $19 \%$ seulement des nuitées induites par les voyages personnels ont eu pour cadre un pays étranger ou les DOM 
(DGE, 2018). C'est aussi le cas lorsque des non-résidents ont acquis, grâce à des séjours fréquents, des compétences poussées - pas nécessairement dans le même lieu, car une partie des savoirs peut être réinvestie ailleurs. Dans ce cas, une grande partie des besoins sont satisfaits de façon autoproduite par les touristes, notamment l'alimentation, le transport, voire même l'hébergement. L'estimation du volume d'affaires est d'ailleurs difficile à établir car la familiarité favorise l'économie informelle.

17 Entre ces deux situations extrêmes, une configuration intermédiaire peut être définie, qui associe la chaîne de valeur traditionnelle pour les fonctions de transport et d'hébergement et une constellation au sein de laquelle les individus piochent selon leurs projets.

Cette première approche préalable consiste donc à identifier les combinaisons de types de systèmes d'acteurs présents.

\subsection{Mobilisation de la typologie des lieux}

Une seconde approche aborde la question par les lieux. Dans leur analyse, Durieux et al. (2015) esquissent une telle démarche en distinguant les effets du tourisme sur l'emploi selon différents types d'espace. Leur analyse est cependant élaborée à partir d'une approche classique, fondée sur les distinctions géographiques entre littoral et montagne, d'une part, et entre ville et campagne, d'autre part. Elle est certes un peu sophistiquée par l'introduction de distinction selon les tailles de ville (unité urbaine parisienne, grandes unités urbaines, unités urbaines moyennes), l'altitude (haute montagne, moyenne montagne) ou les littoraux (méditerranéen, corse, atlantique, breton). Mais cette approche tend à naturaliser les distinctions. Par ailleurs, la notion de station est mobilisée mais sans que les auteurs n'explicitent ce dont il s'agit et en limitant son application à la seule montagne.

20 Une approche plus fine est nécessaire (tableau 2), qui mobilise une typologie des lieux (Équipe MIT, 2002 ; Duhamel, 2018). En effet, dans les lieux créés ou inventés par le tourisme, il est possible de considérer que l'essentiel des activités est animé par le tourisme et donc près de $100 \%$ des emplois peuvent y être affectés. Il s'agit des lieux créés par le tourisme, comme les comptoirs ou les stations. Toutefois, ces dernières constituent également des lieux de vie, donc la part du tourisme dans l'emploi y est moindre, de l'ordre de 80 à $90 \%$. Au-delà, les stations littorales ont fortement évolué depuis les années 1970-1980 avec des processus de diversification économique, notamment en matière de résidentialisation, à savoir l'accueil de populations, notamment retraitées, qui choisissent d'habiter à l'année dans les lieux autrefois nettement dominés par le tourisme. Cette évolution se produit également pour des actifs qui s'installent dans des stations touristiques dès lors que la croissance de la mobilité contemporaine, dans une société à individus mobiles (Stock, 2005), permet d'accéder quotidiennement à des emplois. Il faudrait donc ajuster en fonction de la dynamique démographique locale.

21 Dans les lieux investis, deux cas peuvent être distingués : d'une part, ceux dans lesquels le tourisme constitue le principal moteur économique (sites - lieux que les touristes visitent mais dans lesquels ils ne dorment pas; villages ou villes touristifiés - lieux historiques subvertis par le tourisme, devenu moteur économique principal, comme Cordes ou Venise). D'autre part, dans les villes touristiques, le tourisme est un secteur 
parmi d'autres, il faudrait donc estimer son poids et déterminer les branches entraînées.

Tableau 2. Part des emplois touristiques selon les différents types de lieu

\begin{tabular}{|l|l|l|l|}
\hline Type & Sous-type & Caractéristiques principales & Mesure des emplois \\
\hline \multirow{2}{*}{$\begin{array}{l}\text { Lieux créés ou } \\
\text { inventés par le } \\
\text { tourisme }\end{array}$} & Comptoir & $\begin{array}{l}\text { Lieux fermés gérés par un seul } \\
\text { opérateur }\end{array}$ & $100 \%$ \\
\cline { 2 - 5 } & Station & $\begin{array}{l}\text { Lieux créés par le tourisme mais } \\
\text { ouvert }\end{array}$ & 80 à $90 \%$ \\
\cline { 2 - 5 } & Station ville & $\begin{array}{l}\text { Idem mais processus de de } \\
\text { diversification en cours }\end{array}$ & $\begin{array}{l}60 \text { à } 80 \% \text { selon l'intensité } \\
\text { de la diversification }\end{array}$ \\
\hline \multirow{2}{*}{$\begin{array}{l}\text { Lieux investis par le } \\
\text { tourisme }\end{array}$} & $\begin{array}{l}\text { Ville } \\
\text { touristique }\end{array}$ & $\begin{array}{l}\text { Le tourisme est un secteur } \\
\text { économique parmi d'autres }\end{array}$ & $\begin{array}{l}10 \text { à } 15 \% \text { selon } \\
\text { touristicité }\end{array}$ \\
\cline { 2 - 5 } & Ville étape & $\begin{array}{l}\text { Idem mais la fonction } \\
\text { d'hébergement domine }\end{array}$ & $10 \%$ \\
\cline { 2 - 5 } & $\begin{array}{l}\text { Ville } \\
\text { touristifiée }\end{array}$ & $\begin{array}{l}\text { Le tourisme est devenu la } \\
\text { fonction principale }\end{array}$ & 80 à $90 \%$ \\
\hline
\end{tabular}

Il faudrait ensuite (tableau 3) combiner les deux approches car les comportements des touristes ne sont pas homogènes. Ainsi, dans un même lieu, situé le long du littoral en France, des touristes relativement autonomes, par exemples des résidents dotés de fortes dispositions et compétences, côtoient d'autres touristes, davantage hétéronomes du fait de leur confrontation à un degré plus ou moins élevé d'altérité, notamment les non-résidents, qui les porte à privilégier la mobilisation de la chaîne de valeur, ou des résidents qui font le choix d'un séjour tout compris qui correspond mieux à leur projet du moment.

Tableau 3. Croisement entre le comportement des touristes et les types de lieux

\begin{tabular}{|l|l|l|l|}
\hline & $\begin{array}{l}\text { Forte } \\
\text { altérité }\end{array}$ & $\begin{array}{l}\text { Altérité } \\
\text { intermédiaire }\end{array}$ & $\begin{array}{l}\text { Forte } \\
\text { familiarité }\end{array}$ \\
\hline Station côte atlantique française & & $10 \%$ & Dominante $90 \%$ \\
\hline $\begin{array}{l}\text { Station haute montagne très } \\
\text { renommée }\end{array}$ & $40 \%$ & $30 \%$ & $30 \%$ \\
\hline Paris & $20 \%$ & $30 \%$ & $50 \%$ \\
\hline
\end{tabular}

Enfin, il faudrait également tenir compte de la saisonnalité afin de pondérer les emplois en équivalent temps-plein. 
24 La piste que nous proposons consiste donc à enquêter auprès des touristes pour connaître leurs pratiques réelles en matière de satisfaction de leurs besoins. Ensuite, une estimation du volume d'affaires réalisé par des transactions touristiques au sens stricte et de manière expérimentale, et non sur une base conventionnelle peu discutée, permettrait d'évaluer la part du tourisme dans les activités présentes dans les lieux et de déduire le nombre d'emploi au prorata entre les achats effectués par les touristes et ceux effectués par les habitants.

\section{Conclusion}

Une telle approche serait certes minutieuse, mais elle ne présente pas de difficultés insurmontables. Il suffit peut-être, pour être réaliste, de renoncer à vouloir produire des données annuellement, pour un résultat assez peu intéressant tant les variations d'une année sur l'autre sont minimes. Sortir de l'illusion et des faux-semblants nous semble mériter une approche un peu plus construite, même si elle se révèle relativement longue à mettre en œuvre.

\section{BIBLIOGRAPHIE}

Laurent BOTTI, Nicolas PEYPOCH et Bernardin SOLONANDRASANA, Économie du tourisme, coll. « Les topos », Dunod, 2013.

Jean-Louis CAсCомо (dir.), Fondements d'économie du tourisme. Acteurs, marchés, stratégies, coll. « Les métiers du tourisme », De Boeck, 2007.

Cécile CLERGEAU, « Création de richesses par le tourisme », dans Philippe VIOLIER (dir.), Le tourisme, un phénomène économique, coll. « Études », La Documentation française, 2013.

DIRECTION GÉNÉRALE DES ENTREPRISES, Mémento du tourisme, parution annuelle.

DIRECTION GÉNÉRALE DES ENTREPRISES, Les chiffres clés du tourisme, parution annuelle.

Philippe DUHAMEL, Géographie du tourisme et des loisirs. Dynamiques, acteurs, territoires, coll. « U », Armand Colin, 2018.

Stéphanie DURIEUX, Pascal EUSEBIO et David LÉVY, « Un million d'emplois liés à la présence de touristes. La moitié dans les espaces urbains », Insee Première, n 1555, juin 2015.

ÉQUIPE MIT, Tourismes 1. Lieux communs, coll. « Mappemonde », Belin, 2002.

Rémy KNAFOU et Mathis STOCK, « Tourisme », dans Jacques LÉVY et Michel LUSSAULT, Dictionnaire de la géographie et de l'espace des sociétés, Belin, 2003.

Joël RABOTEUR, Introduction à l'économie du tourisme, L'Harmattan, 2000.

Thea M. SINCLAIR et Michael John STABLER, The economics of tourism, Routledge, 1997.

Mathis STOCK, «Les sociétés à individus mobiles : vers un nouveau mode d'habiter ?", EspacesTemps.net, 2005 [https://www.espacestemps.net/articles/societes-individus-mobiles/]. 
Mathis STOCK, Vincent coËFfÉ et Philippe VIOLIER, avec la collaboration de Philippe DUHAMEL, Les enjeux contemporains du tourisme. Une approche géographique, Presses universitaires de Rennes, 2017.

François VELLAS, Économie et politique du tourisme international, Economica, 2006.

\section{NOTES}

1. Lorsque la démonstration nous oblige à mobiliser la définition ou l'appellation « tourisme » au sens de l'OMT et des institutions, beaucoup trop large pour nous, le mot apparaît entre guillemets.

2. Selon Les chiffres clés du tourisme en France, édition 2018, autre publication de la DGE.

3. Cf. site internet : https://www.tourisme-territoires.net/rn2d/

\section{RÉSUMÉS}

Cet article analyse, dans un premier temps, les statistiques sur l'emploi touristique publiées par la direction générale des Entreprises du ministère de l'Économie et des Finances. Elles sont fondées sur une catégorie dite des "activités caractéristiques » qui isole certaines branches de l'économie, lesquelles, dans la réalité, fournissent des services également consommés par les habitants, comme la restauration et les cafés. Par ailleurs, cette mesure n'est pas stable dans le temps. Ensuite, nous proposons une contribution pour une approche plus fine qui se fonde sur les pratiques des touristes et sur une typologie des lieux et espaces touristiques.

This paper analyses firstly the tourist employment statistics published by the Directorate General for Enterprise (D.G.E) of the French Ministry of Economy and Finance. Such statistics are based upon a category named "typical activities" that isolates certain sectors of the economy, which in reality provide services consumed by local inhabitants, for instance restaurants and cafés. Furthermore, such a measurement is not stable over time. Secondly, we propose here a contribution to a much more precise approach based upon the tourist practices and a typology of tourist places and spaces.

\section{INDEX}

Mots-clés : statistique, emploi, consommation, tourisme

Keywords : statistics, employment, consumption, tourism

\section{AUTEUR}

\section{PHILIPPE VIOLIER}

Géographie, Université d'Angers, Esthua, UM ESO

philippe.violier@univ-angers.fr 